\title{
Decoding Parkinson Disorder (PD) Pathophysiology: $\alpha$-Synuclein over-expression enhances inward calcium influx and exosome release in LUHMES cells. \\ Rohit Kumar, ${ }^{1}$ Günter U. Höglinger, ${ }^{1,}, 2$ Thomas Kögelsperger, ${ }^{1,3}$
}

\begin{abstract}
${ }^{1}$ German Center for Neurodegenerative Diseases e.V. (DZNE), Dept. for Translational Neurodegeneration, D-81377 Munich, Germany, ${ }^{2}$ Klinikum rechts der Isar der Technischen Universität München. ${ }^{3}$ Neurologische Klinik und Poliklinik, Klinikum der Universität München, Ludwig-MaximiliansUniversität, Marchioninistraße 15, 81377, München
\end{abstract}

\section{Introduction:}

The hall-mark events for Parkinson's Disorder (PD) are Lewy Body (LB) formation and further degeneration of midbrain dopaminergic neurons (Li et al., 2008). Even though $\alpha$-Synuclein (SNCA) is established as key molecular component of LBs, yet there is no clear understanding of pathophysiological mechanisms acting behind the scene. In such scenario we failed to comprehensively demonstrate the exact reasons behind PD pathology seeding and its spread. At present there are several mechanisms proposed for PD pathology seeding and spreading (Angelova et al., 2016) among these the transfer of pathological SNCA via exosomal/extracellular vesicles release is also advocated. This motivated us to investigate such mechanism.

\section{Methods:}

We use patch-clamp electrophysiology (B. Sakmann \& Neher, 1984) on fetal human mesencephalic cell line (LUHMES, Lund human mesencephalic); which is the model system to mimic the dopaminergic neurons in vitro. Recordings from LUHMES cells overexpressing SNCA were made using a whole cell patch in voltage clamp and current clamp mode in order to measure the calcium currents $\left(\mathbf{I}_{c a}\right)$ and membrane capacitance $(\mathbf{C m})$. Further assessment of the media collected from SNCA overexpressing cells was done by using Nano Particle Tracking Analysis (NTA)

\section{Results:}

In early stage experiments overexpression of SNCA has led to the subtle morphophysiological changes to LUHMES cells. An enhanced inward $\mathbf{I}_{c a}$ and membrane capacitance $(\mathbf{C m})$ was observed. NTA results suggest us a higher particle concentration for the size range between 50-150 $\mathrm{nm}$ which corresponds to the size of exosomes.

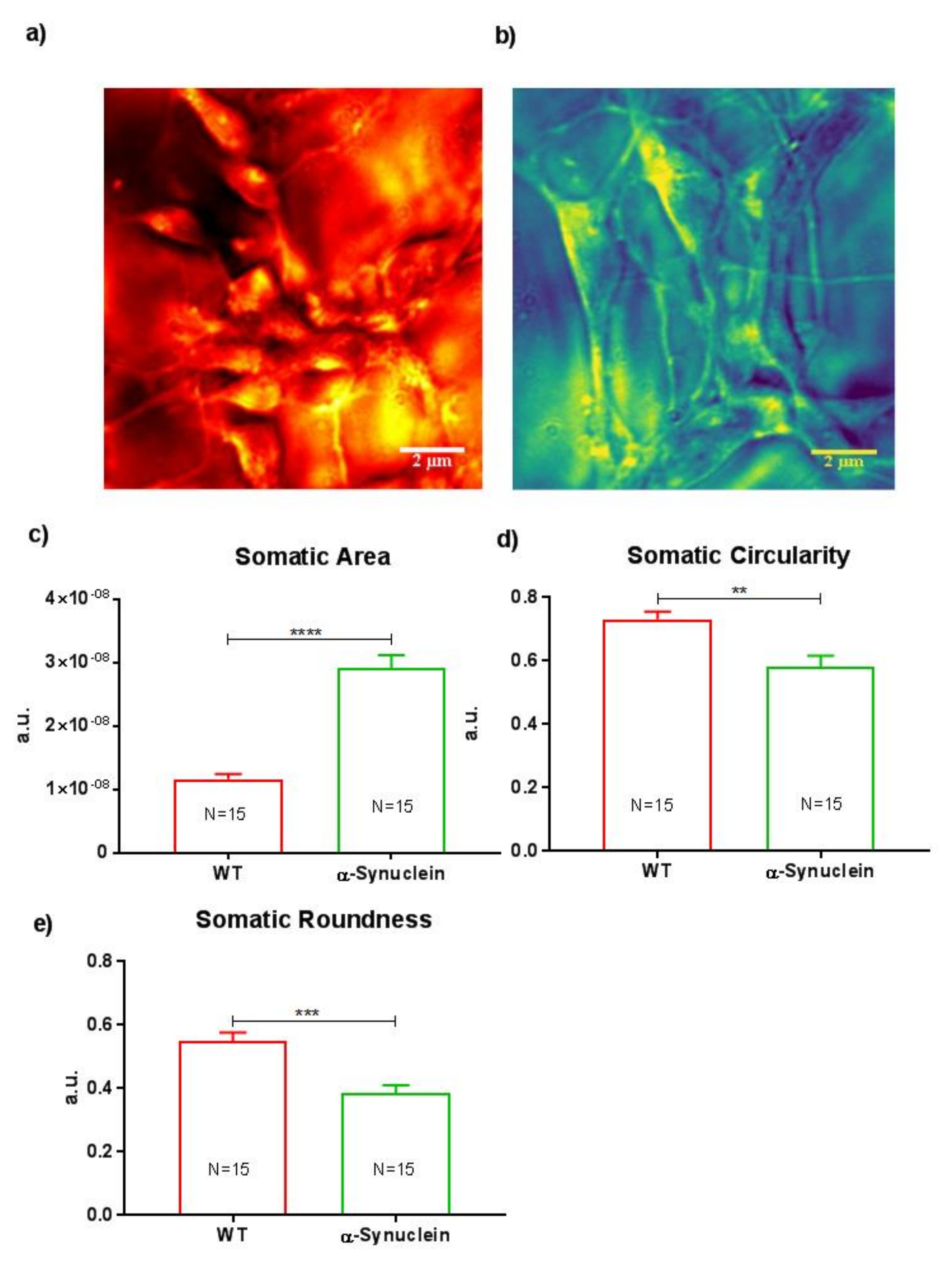

Fig.1. SNCA overexpression induces morphological changes in LUHMES cells.
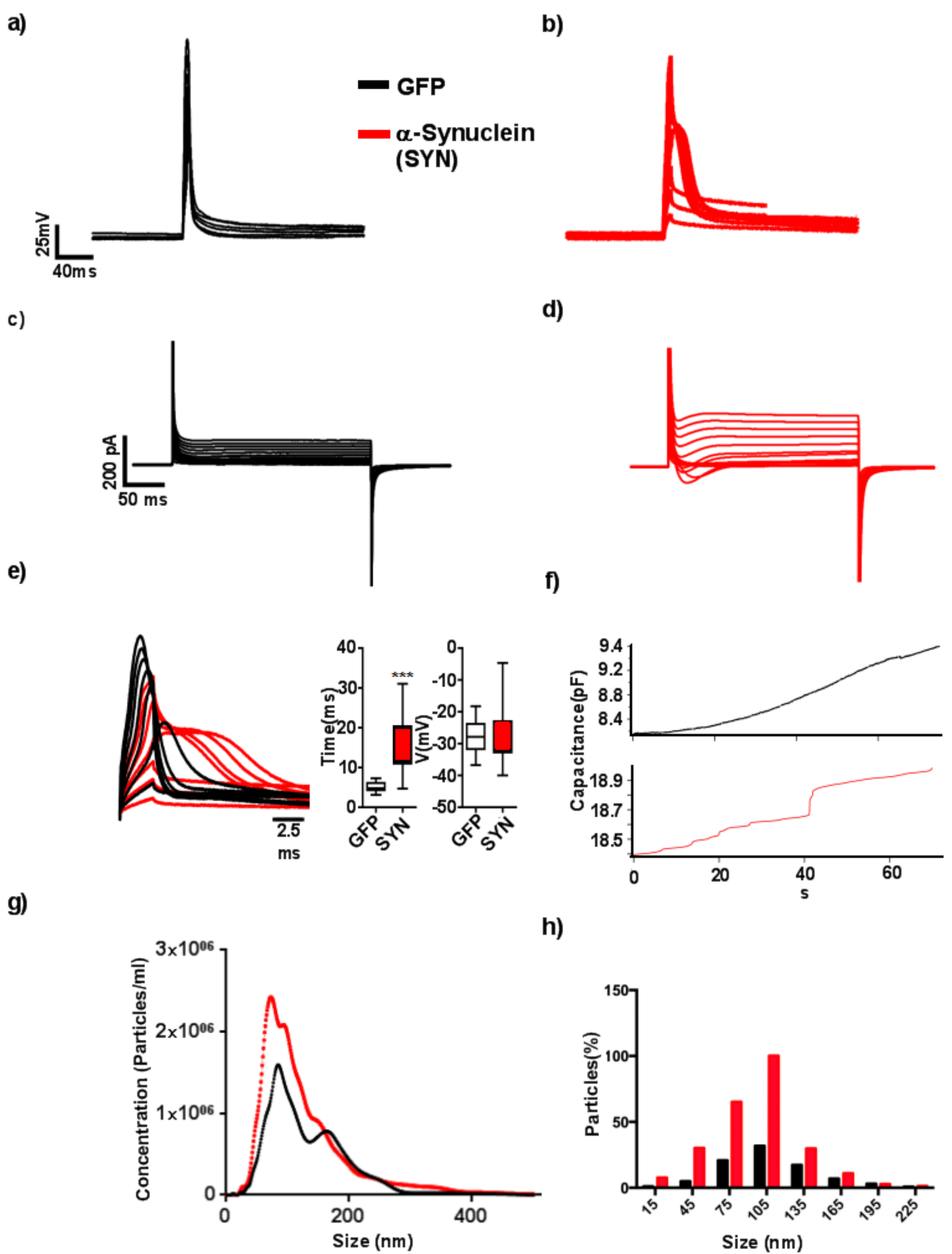

Fig.2. SNCA over-expressing cells experience Physiological changes as a strong pharmacologically isolated calcium influx facilitating exosome release.

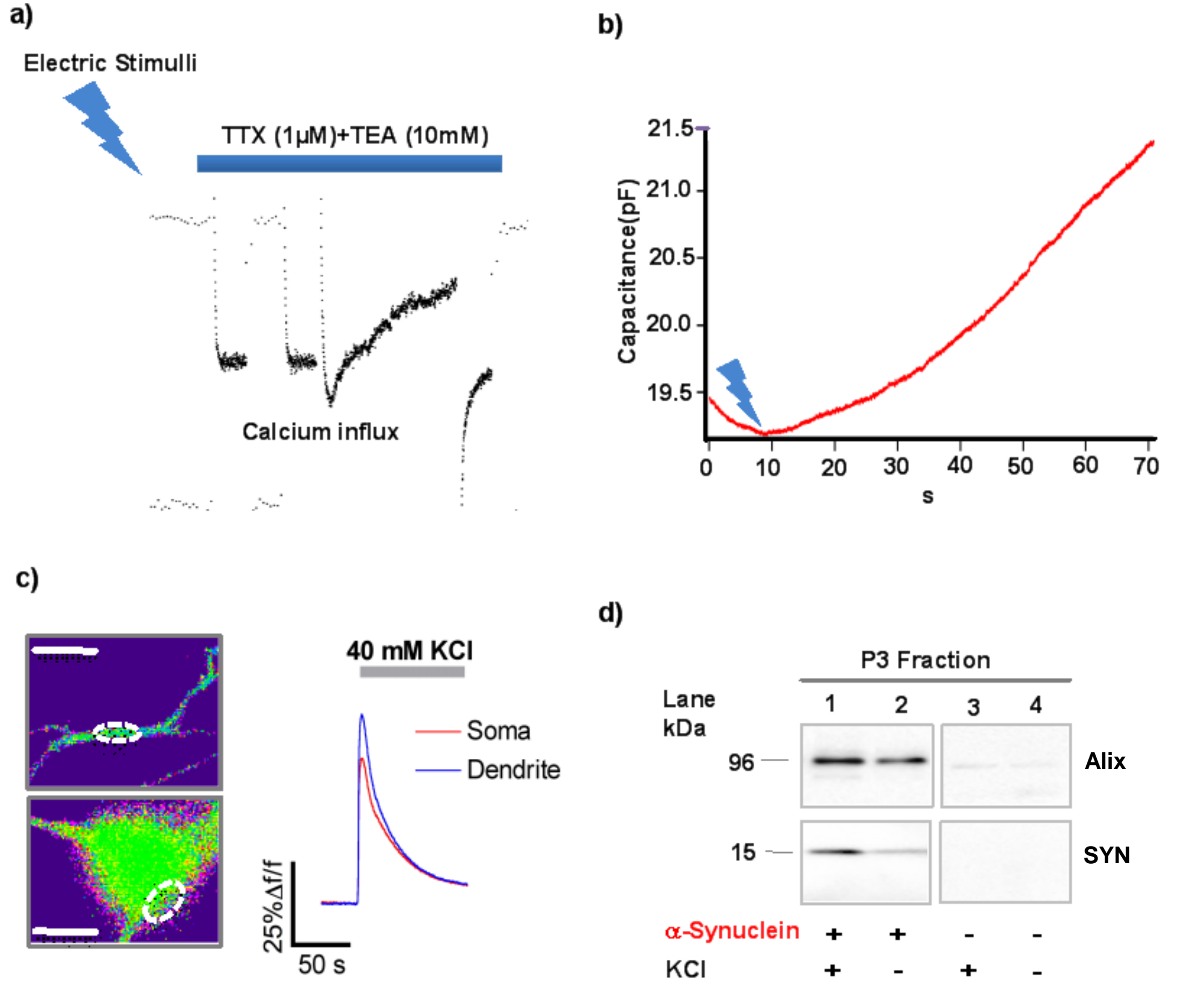

Fig.3. Inward calcium influx linearly correlates to the release of available exosomes.

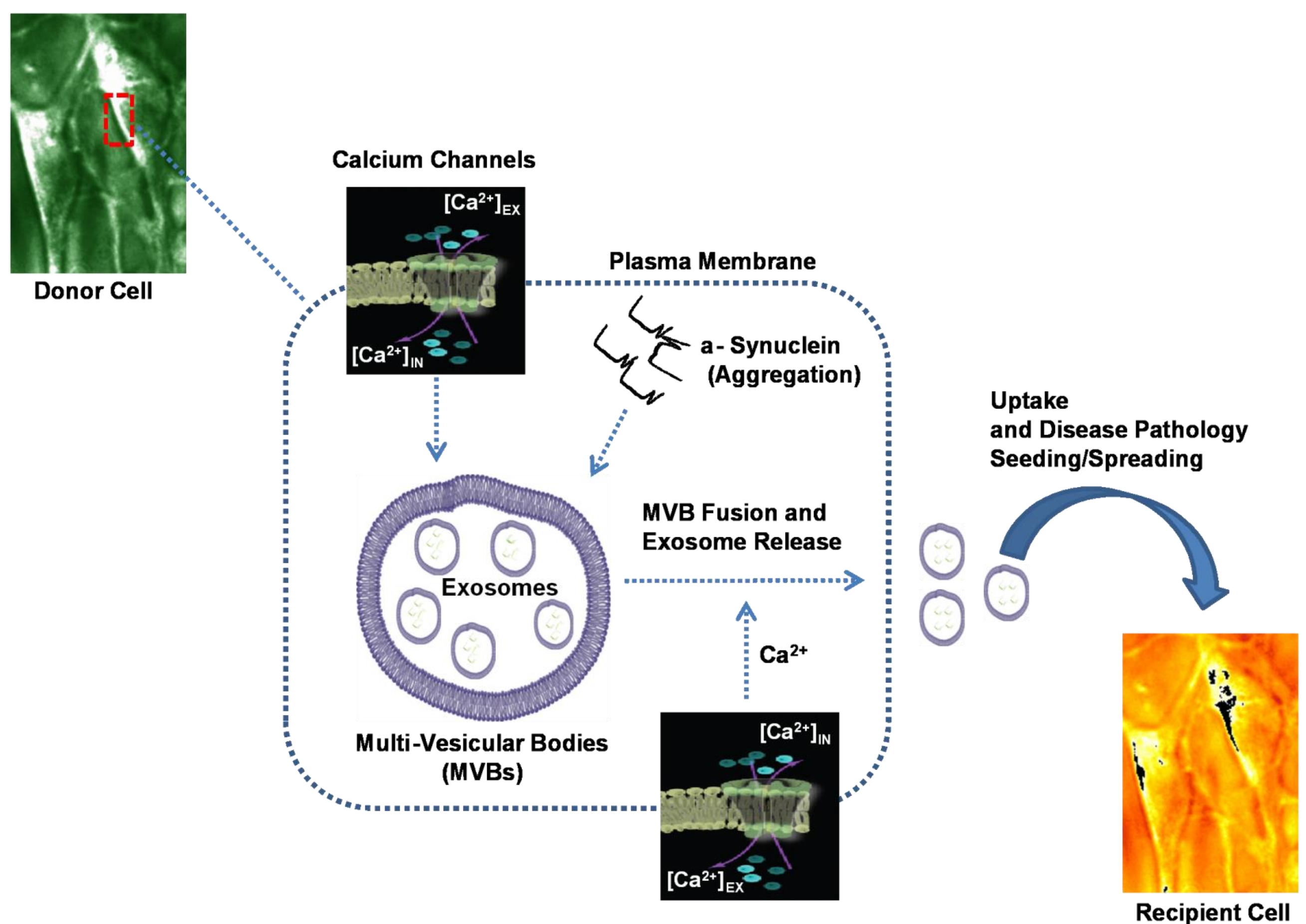

Fig.4. Possible exosomal role and physiological events of PD pathology seeding and spreading

\section{Conclusions:}

These finding allow us to anticipate a role of calcium ion in formation of a pathophysiological state supplemented via excess production of exosomes and thus provide us initial glimpse of plausible physiological machinery responsible for the disease pathology onset and further propagation.

Acknowledgements: All authors are thankful to ParkinsonFon'ds (https://www.parkinsonfonds.de/uber-uns/ ) for funding this research project. First author is also thankful to Deutsches Zentrum für Neurodegenrative Erkrankungen (DZNE) (https://www.dzne.de/ ) for supporting my participation to this event and the fellow lab members for their support

\section{Reference:}

Angelova, P. R., Ludtmann, M. H., Horrocks, M. H., Negoda, A., Cremades, N., Klenerman, D., .. . Abramov, A. Y. (2016). Ca2+ is a key factor in alphasynuclein- induced neurotoxicity. J Cell Sci, 129(9), 1792-1801. doi: $10.1242 /$ jcs. 180737

B. Sakmann, \& Neher, E. (1984). Patch Clamp Technique For Studying Ionic Channels. In Excitable Membranes. Annual Reviews Physiology, 46, 455-472.

Li, J. Y., Englund, E., Holton, J. L., Soulet, D., Hagell, P., Lees, A. J., . . Brundin, P. (2008). Lewy bodies in grafted neurons in subjects with Parkinson's disease suggest host-to-graft disease propagation. Nat Med, 14(5), 501-503. doi:10.1038/nm1746 